\title{
苯乙酮基吡啶季铵盐与醇的反应
}

\author{
余川 周洁陈慧刘绪峰张万轩*
}

(湖北大学有机化工新材料湖北省协同创新中心 有机功能分子合成与应用教育部重点实验室 武汉 430062)

\begin{abstract}
摘要 苯乙酮基吡啶季铵盐具有多种反应活性, 但它带有的羰基的反应性很少引起人们的关注. 研究发现苯乙酮基吡 啶季铵盐在碱性条件下与醇(或酚)反应时，吡啶基带着 $\alpha$-碳原子离去，生成相应的苯甲酸酯类物质. 与醇反应时产率较 高( $80 \% \sim 98 \%)$, 与苯酚反应时, 产率为 $48 \%$.

关键词 苯乙酮基吡啶季铵盐; 醇; 苯甲酸酯衍生物
\end{abstract}

\section{Reactions of Acetophenonyl Pyridinium Salts with Alcohols}

\author{
She, Chuan Zhou, Jie Chen, Hui Liu, Xufeng Zhang, Wanxuan* \\ (Hubei Collaborative Innovation Center for Advanced Organic Chemical Material, Key Laboratory for the Synthesis and Ap- \\ plication of Organic Functional Molecules, Ministry of Education, Hubei University, Wuhan 430062)
}

\begin{abstract}
Acetophenonyl pyridinium salts are versatile reagents in organic reactions, but the reactivities of their carbonyls were seldomly studied. It was found that the reactions of the carbonyls with alcohols occurred when acetophenonyl pyridinium salts were treated with alcohols (or phenol) in the presence of a base giving rise to benzoates, and the departure of pyridyl with the $\alpha$-carbon atom was involved. High yields $(80 \% \sim 98 \%)$ were achieved when they reacted with alcohols, however a yield of $48 \%$ were obtained when phenol was used instead of alcohols.
\end{abstract}

Keywords acetophenonyl pyridinium salt; alcohol; derivative of benzoate

吡啶季铵盐是重要的有机合成试剂, 它可以发生亲 核加成、Michael 加成、1,3-偶极加成、亲核取代和 $\sigma$ 迁移重排等反应. 吡啶季铵盐之所以具有如此丰富的化 学性质, 是因为正电荷使吡啶环活化, 其 2-, 4-, 6-位更 易被亲核进攻; 此外, 吡啶季铵盐上与氮原子相连的亚 甲基失去氢后, 可以形成一种重要的活性中间体一一氮 叶立德, 因此它也可以作为亲核试剂应用于有机合成 中 $^{[1]}$. 在苯乙酮吡啶季铵盐中, 与吡啶氮原子相连的亚 甲基同时与一个羰基相连，因此更容易形成氮叶立德， 被应用于合成多种含氮杂环化合物, 如用于合成吡 啶 ${ }^{[2]}$ 、吲嗪 ${ }^{[3]}$ 、嘧啶 ${ }^{[4]}$ 衍生物等. 在这些反应中, 苯乙酮 吡啶季铵盐主要提供亲核性的碳负离子. 事实上, 其羰 基也能和一些亲核性试剂反应，但有关报道较少 ${ }^{[5]}$. 我 们研究发现, 在碱性条件下, 苯乙酮基吡啶季铵盐中的 羰基很容易与醇发生反应生成苯甲酸酯类物质(Eq. 1). 在此反应中, 苯乙酮基吡啶季铵盐表现出独特的性质:
反应中吡啶基带着一个碳原子离去, 使苯乙酮基失去一 个碳原子，最终生成苯甲酸酯类物质. 我们对该反应的 合适条件进行了探讨, 并研究了苯乙酮基吡啶季铵盐和 多种醇的反应情况.

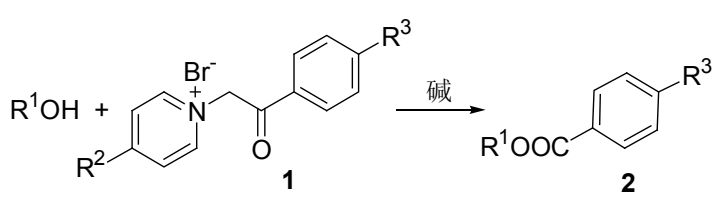

\section{1 结果与讨论}

\section{1 季铵盐与苯甲醇的反应}

首先以苯甲醇和季铵盐 $1 \mathrm{a}$ 的反应为例, 对反应条 件进行了探讨, 发现以甲苯为溶剂, $\mathrm{Na}_{2} \mathrm{CO}_{3}$ 作为碱, 在 回流条件下反应，产物苯甲酸芐酯的产率最高. 反应物 以 $n(\mathbf{1 a}): n$ (苯甲醇) $: n\left(\mathrm{Na}_{2} \mathrm{CO}_{3}\right)=1: 1.4: 3.5$ 为宜. 在 此条件下进一步研究了季铵盐 $1 \mathrm{~b} \sim 1 \mathrm{e}$ 与苯甲醇的反应

\footnotetext{
*E-mail: zhangwx@hubu.edu.cn

Received June 23, 2014; revised August 8, 2014; published online August 28, 2014.

Project supported by the National Natural Science Foundation of China (No. 20872031) and the Key Laboratory for the Synthesis and Application of Organic Functional Molecules, Ministry of Education.

国家自然科学基金(No. 20872031)和有机功能分子合成与应用教育部重点实验室资助项目.
} 
(表 1). 从表 1 可以看出, 当季铵盐的吡啶环对位连有推 电子基甲基时(表 1, Entries 2,5), 比同类的其它季铵盐 (吡啶环对位连有吸电子的氰基或无取代基)产率高; 而 苯乙酮基的苯环对位连有吸电子硝基时，反应产率较低 (表 1, Entry 3 vs Entry 1; Entry 5 vs Entry 2).

表 1 苯甲醇与多种季铵盐反应的结果 ${ }^{a}$

Table 1 Results for the reactions of benzyl alcohol with acetophenonyl pyridinium salts

\begin{tabular}{cllcc}
\hline Entry & \multicolumn{1}{c}{ 季铵盐 $\mathbf{1}$} & \multicolumn{1}{c}{ 产物 $\mathbf{2}$} & 反应时间 $/ \mathrm{h}$ 产率 $/ \%$ \\
\hline 1 & $\mathbf{1 a}: \mathrm{R}^{2}=\mathrm{R}^{3}=\mathrm{H}$ & $\mathbf{2 a}: \mathrm{R}^{3}=\mathrm{H}$ & 15 & 90 \\
2 & $\mathbf{1 b}: \mathrm{R}^{2}=\mathrm{CH}_{3}, \mathrm{R}^{3}=\mathrm{H}$ & $\mathbf{2 a}: \mathrm{R}^{3}=\mathrm{H}$ & 10 & 98 \\
3 & $\mathbf{1 c}: \mathrm{R}^{2}=\mathrm{H}, \mathrm{R}^{3}=\mathrm{NO}_{2}$ & $\mathbf{2 b}: \mathrm{R}^{3}=\mathrm{NO}_{2}$ & 16 & 82 \\
4 & $\mathbf{1 d}: \mathrm{R}^{2}=\mathrm{CN}, \mathrm{R}^{3}=\mathrm{NO}_{2}$ & $\mathbf{2 b}: \mathrm{R}^{3}=\mathrm{NO}_{2}$ & 13 & 85 \\
5 & 1e: $\mathrm{R}^{2}=\mathrm{CH}_{3}, \mathrm{R}^{3}=\mathrm{NO}_{2}$ & $\mathbf{2 b}: \mathrm{R}^{3}=\mathrm{NO}_{2}$ & 15 & 95 \\
\hline$a$ 反应条件: $n(\mathbf{1}): n$ (苯甲醇) $: n\left(\mathrm{Na}_{2} \mathrm{CO}_{3}\right)=1: 1.4: 3.5$, 甲苯中回流.
\end{tabular}

\section{2 多种季铵盐与多种醇的反应}

根据以上研究结果, 利用对甲基吡啶制备的季铵盐 $1 \mathrm{~b}, 1 \mathrm{e}, 1 \mathrm{f}, 1 \mathrm{~g}$ 分别与多种醇或苯酚反应, 得到相应的苯 甲酸酯类衍生物(表 2). 从表 2 可以看出, 与醇反应时, 产率都很高，达到 $80 \%$ ～98\%. 当同一种醇与不同季铵 盐反应时，季铵盐苯乙酮基的苯环上带有吸电子基团 (如硝基、氯)时，产率稍低; 而连有给电子基(甲氧基)时， 产率较高. 另一方面, 当同一种季铵盐与不同醇反应时, 伯醇的产率高于仲醇, 这可能是由于仲醇的位阻较大. 1e 与苯酚反应时, 产率最低, 只有 $48 \%$ (表 2, Entry 12).

\section{3 反应机理的探讨}

文献[5]表明，当苯乙酮基吡啶季铵盐(1a)在高浓度 的碱性水溶液中, 会生成苯甲酸(盐), 从所得到的产物 看, 苯乙酮基吡啶季铵盐与醇的反应机理可能与水的反 应机理相似，但文献没有阐明反应机理 ${ }^{[5]}$. 根据羰基的 反应性, 以及所生成的主要产物, 推测此反应可能按如 下机理进行 (Scheme 1): 即在碱协助下, 醇的氧原子进
攻季铵盐 1 的羰基，发生亲核加成反应，然后吡啶基带 着 $\alpha$-碳原子离去，得到酯 2 和活泼中间体一一叶立德 3 . 由于该反应有多种副产物，且难以分离、鉴定，实验中 未能确认反应中生成化合物 $\mathbf{3}$ 的衍生物，所以该反应机 理有待进一步研究.

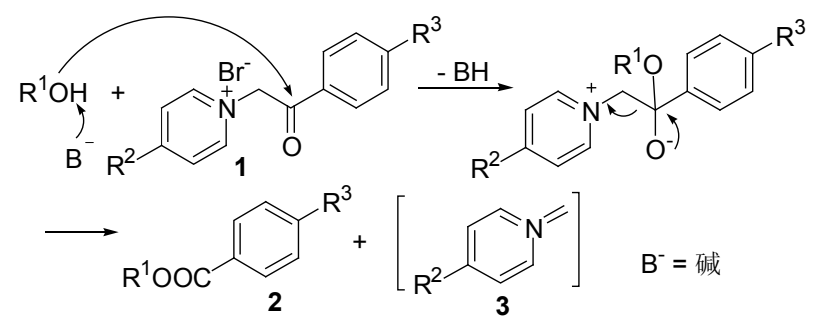

Scheme 1

\section{2 结论}

典型的酮与醇反应一般生成缩酮或半缩酤，而苯乙 酮基季铵盐与醇(或酚)在碱性条件下反应时，表现出独 特的性质：吡啶基带着一个碳原子离去而生成苯甲酸酯 类物质，产率为中等或较高( $48 \% \sim 98 \%)$.

\section{3 实验部分}

\section{1 仪器与试剂}

红外光谱仪: PE-Spectrum One 型红外光谱仪, $\mathrm{KBr}$ 压片; 核磁共振仪: INOVA (600 MHz)或 WIPM (400 $\mathrm{MHz})$, 以 $\mathrm{CDCl}_{3}$ 或気代丙酮为溶剂, TMS 为内标.

苯乙酮基季铵盐为自制; 吡啶用 $\mathrm{KOH}$ 干燥后重蒸; 乙腈(A.R.)在氢化钻中回流 $3 \mathrm{~h}$ 以上再收集使用. 苯酚经 重结晶后再用. 其它试剂和溶剂未经处理, 直接使用.

\section{2 实验方法}

\subsection{1 苯乙酮基季铵盐的制备}

取一干燥的 $250 \mathrm{~mL}$ 三颈烧瓶, 加入 $0.03 \mathrm{~mol} 2$-溴 苯乙酮(或其它苯环取代的 2-溴苯乙酮)、0.02 $\mathrm{mol}$ 吡啶

表 2 多种醇与多种苯乙酮基吡啶季铵盐反应的结果 ${ }^{a}$

Table 2 Results for the reactions of alcohols with acetophenonyl pyridinium salts

\begin{tabular}{|c|c|c|c|c|}
\hline Entry & 醇 & 季铵盐 1 & 产物 2 & 产率 $/ \%$ \\
\hline 1 & $\mathrm{PhCH}_{2} \mathrm{OH}$ & 1b: $\mathrm{R}^{2}=\mathrm{CH}_{3}, \mathrm{R}^{3}=\mathrm{H}$ & 2a: $\mathrm{R}^{1}=\mathrm{PhCH}_{2}, \mathrm{R}^{3}=\mathrm{H}$ & 98 \\
\hline 2 & $\mathrm{PhCH}_{2} \mathrm{OH}$ & 1e: $\mathrm{R}^{2}=\mathrm{CH}_{3}, \mathrm{R}^{3}=\mathrm{NO}_{2}$ & 2b: $\mathrm{R}^{1}=\mathrm{PhCH}_{2}, \mathrm{R}^{3}=\mathrm{NO}_{2}$ & 85 \\
\hline 3 & $\mathrm{PhCH}_{2} \mathrm{CH}_{2} \mathrm{OH}$ & 1b: $\mathrm{R}^{2}=\mathrm{CH}_{3}, \mathrm{R}^{3}=\mathrm{H}$ & 2c: $\mathrm{R}^{1}=\mathrm{PhCH}_{2} \mathrm{CH}_{2}, \mathrm{R}^{3}=\mathrm{H}$ & 90 \\
\hline 4 & $\mathrm{PhCH}_{2} \mathrm{CH}_{2} \mathrm{OH}$ & 1e: $\mathrm{R}^{2}=\mathrm{CH}_{3}, \mathrm{R}^{3}=\mathrm{NO}_{2}$ & 2d: $\mathrm{R}^{1}=\mathrm{PhCH}_{2} \mathrm{CH}_{2}, \mathrm{R}^{3}=\mathrm{NO}_{2}$ & 84 \\
\hline 5 & $p-\mathrm{NO}_{2} \mathrm{C}_{6} \mathrm{H}_{4} \mathrm{CH}_{2} \mathrm{OH}$ & 1b: $\mathrm{R}^{2}=\mathrm{CH}_{3}, \mathrm{R}^{3}=\mathrm{H}$ & 2e: $\mathrm{R}^{1}=p-\mathrm{NO}_{2} \mathrm{C}_{6} \mathrm{H}_{4} \mathrm{CH}_{2}, \mathrm{R}^{3}=\mathrm{H}$ & 96 \\
\hline 6 & $\mathrm{CH}_{3}\left(\mathrm{CH}_{2}\right)_{3} \mathrm{OH}$ & 1e: $\mathrm{R}^{2}=\mathrm{CH}_{3}, \mathrm{R}^{3}=\mathrm{NO}_{2}$ & 2f: $\mathrm{R}^{1}=\mathrm{CH}_{3}\left(\mathrm{CH}_{2}\right)_{3}, \mathrm{R}^{3}=\mathrm{NO}_{2}$ & 83 \\
\hline 7 & $\mathrm{CH}_{3}\left(\mathrm{CH}_{2}\right)_{3} \mathrm{OH}$ & 1f: $\mathrm{R}^{2}=\mathrm{CH}_{3}, \mathrm{R}^{3}=\mathrm{Cl}$ & 2g: $\mathrm{R}^{1}=\mathrm{CH}_{3}\left(\mathrm{CH}_{2}\right)_{3}, \mathrm{R}^{3}=\mathrm{Cl}$ & 91 \\
\hline 8 & $\mathrm{CH}_{3}\left(\mathrm{CH}_{2}\right)_{3} \mathrm{OH}$ & 1g: $\mathrm{R}^{2}=\mathrm{CH}_{3}, \mathrm{R}^{3}=\mathrm{OCH}_{3}$ & 2h: $\mathrm{R}^{1}=\mathrm{CH}_{3}\left(\mathrm{CH}_{2}\right)_{3}, \mathrm{R}^{3}=\mathrm{OCH}_{3}$ & 95 \\
\hline 9 & $\mathrm{CH}_{3}\left(\mathrm{CH}_{2}\right)_{2} \mathrm{CHOHCH}_{3}$ & 1e: $\mathrm{R}^{2}=\mathrm{CH}_{3}, \mathrm{R}^{3}=\mathrm{NO}_{2}$ & 2i: $\mathrm{R}^{1}=2$-pentyl, $\mathrm{R}^{3}=\mathrm{NO}_{2}$ & 80 \\
\hline 10 & $\mathrm{CH}_{3}\left(\mathrm{CH}_{2}\right)_{2} \mathrm{CHOHCH}_{3}$ & 1f: $\mathrm{R}^{2}=\mathrm{CH}_{3}, \mathrm{R}^{3}=\mathrm{Cl}$ & 2j: $\mathrm{R}^{1}=2$-pentyl, $\mathrm{R}^{3}=\mathrm{Cl}$ & 85 \\
\hline 11 & $\mathrm{CH}_{3}\left(\mathrm{CH}_{2}\right)_{2} \mathrm{CHOHCH}_{3}$ & 1e: $\mathrm{R}^{2}=\mathrm{CH}_{3}, \mathrm{R}^{3}=\mathrm{OCH}_{3}$ & 2k: $\mathrm{R}^{1}=2$-pentyl, $\mathrm{R}^{3}=\mathrm{OCH}_{3}$ & 90 \\
\hline 12 & $\mathrm{PhOH}$ & 1e: $\mathrm{R}^{2}=\mathrm{CH}_{3}, \mathrm{R}^{3}=\mathrm{NO}_{2}$ & 21: $\mathrm{R}^{1}=\mathrm{Ph}, \mathrm{R}^{3}=\mathrm{NO}_{2}$ & 48 \\
\hline
\end{tabular}


(或其它 4-取代吡啶)和 $85 \mathrm{~mL}$ 干燥的乙腈, 加热升温至 $45{ }^{\circ} \mathrm{C}$, 反应过程中用 TLC 跟踪监测反应进程. 反应完 毕后(需要约 $15 \mathrm{~h}$ ), 抽滤收集固体, 用二氯甲烷洗涤固 体, 真空干燥后, 放入干燥器内保存备用. 按此方法分 别制备季铵盐 1a 1g. 产物结构用 ${ }^{1} \mathrm{H}$ NMR 测定，与文 献[6]相符.

\section{2 .2 季铵盐与醇的反应}

反应的一般操作过程: 将一定量醇(酚)、季铵盐 1 、 $\mathrm{Na}_{2} \mathrm{CO}_{3}$ 、溶剂放入反应管中, 在设定的温度下进行反应. 通过 TLC 观察反应进程, 反应结束后, 将反应混合物转 移到圆底烧瓶中, 旋蒸除去溶剂, 剩余物用柱层析分离 提纯. 得到苯甲酸酯类产物 $\mathbf{2 a} \sim \mathbf{2} \mathbf{l}$ (表 2). 产物经 ${ }^{1} \mathrm{H}$ NMR 数据与文献对照确定.

苯甲酸苠酯 $(\mathbf{2 a})^{[7]}$ : ${ }^{1} \mathrm{H} \mathrm{NMR}\left(\mathrm{CDCl}_{3}, 600 \mathrm{MHz}\right) \delta$ : $8.08(\mathrm{~d}, J=7.8 \mathrm{~Hz}, 2 \mathrm{H}), 7.54(\mathrm{t}, J=7.8 \mathrm{~Hz}, 1 \mathrm{H}), 7.45 \sim$ $7.33(\mathrm{~m}, 7 \mathrm{H}), 5.36(\mathrm{~s}, 2 \mathrm{H})$.

4-硝基苯甲酸苠酯(2b) ${ }^{[8]}$ : ${ }^{1} \mathrm{H}$ NMR $\left(\mathrm{CDCl}_{3}, 600\right.$ MHz) $\delta: 8.27$ (d, $J=8.4 \mathrm{~Hz}, 2 \mathrm{H}), 8.23$ (d, $J=8.4 \mathrm{~Hz}, 2 \mathrm{H})$, $7.46(\mathrm{~d}, J=6.6 \mathrm{~Hz}, 2 \mathrm{H}), 7.43 \sim 7.35(\mathrm{~m}, 3 \mathrm{H}), 5.41(\mathrm{~s}, 2 \mathrm{H})$. 苯甲酸苯乙酯 $(2 \mathrm{c}){ }^{[7]}:{ }^{1} \mathrm{H} \mathrm{NMR}\left(\mathrm{CDCl}_{3}, 600 \mathrm{MHz}\right) \delta$ : $7.93(\mathrm{~d}, J=7.2 \mathrm{~Hz}, 2 \mathrm{H}), 7.44$ (t, $J=7.8 \mathrm{~Hz}, 1 \mathrm{H}), 7.32$ (t, $J=7.8 \mathrm{~Hz}, 2 \mathrm{H}), 7.22 \sim 7.13(\mathrm{~m}, 5 \mathrm{H}), 4.44(\mathrm{t}, J=7.2 \mathrm{~Hz}$, 2H), 2.99 ( $\mathrm{t}, J=7.2 \mathrm{~Hz}, 2 \mathrm{H})$.

4-硝基苯甲酸苯乙酯 $(\mathbf{2 d}){ }^{[9]}$ : ${ }^{1} \mathrm{H} \mathrm{NMR}\left(\mathrm{CDCl}_{3}, 400\right.$ MHz) $\delta: 8.18(\mathrm{~d}, J=8.4 \mathrm{~Hz}, 2 \mathrm{H}), 8.09$ (d, $J=8.4 \mathrm{~Hz}, 2 \mathrm{H})$, $7.14 \sim 7.25(\mathrm{~m}, 5 \mathrm{H}), 4.51(\mathrm{t}, J=6.8 \mathrm{~Hz}, 2 \mathrm{H}), 3.02(\mathrm{t}, J=$ $6.8 \mathrm{~Hz}, 2 \mathrm{H})$.

苯甲酸-4-硝基苯甲酯(2e $)^{[10]}$ : ${ }^{1} \mathrm{H}$ NMR $\left(\mathrm{CDCl}_{3}, 600\right.$ $\mathrm{MHz}) \delta: 8.16(\mathrm{~d}, J=8.4 \mathrm{~Hz}, 2 \mathrm{H}), 8.01(\mathrm{t}, J=8.4 \mathrm{~Hz}, 2 \mathrm{H})$, $7.50 \sim 7.56(\mathrm{~m}, 3 \mathrm{H}), 7.37 \sim 7.43(\mathrm{~m}, 2 \mathrm{H}), 5.38(\mathrm{~s}, 2 \mathrm{H})$.

4-硝基苯甲酸正丁酯(2f) ${ }^{[11]}:{ }^{1} \mathrm{H} \mathrm{NMR}\left(\mathrm{CDCl}_{3}, 400\right.$ $\mathrm{MHz}) \delta: 8.27$ (d, $J=7.6 \mathrm{~Hz}, 2 \mathrm{H}), 8.20$ (d, $J=7.6 \mathrm{~Hz}, 2 \mathrm{H})$, $4.38(\mathrm{t}, J=6.4 \mathrm{~Hz}, 2 \mathrm{H}), 1.85 \sim 1.74(\mathrm{~m}, 2 \mathrm{H}), 1.52 \sim 1.44$ $(\mathrm{m}, 2 \mathrm{H}), 0.99(\mathrm{t}, J=7.6 \mathrm{~Hz}, 3 \mathrm{H})$.

4-氯苯甲酸正丁酯 $(\mathbf{2 g})^{[10]}$ : ${ }^{1} \mathrm{H}$ NMR (400 MHz, $\left.\mathrm{CDCl}_{3}\right) \delta: 7.98(\mathrm{~d}, J=8.4 \mathrm{~Hz}, 2 \mathrm{H}), 7.41(\mathrm{~d}, J=8.4 \mathrm{~Hz}$, $2 \mathrm{H}), 4.33(\mathrm{t}, J=6.4 \mathrm{~Hz}, 2 \mathrm{H}), 1.71 \sim 1.81(\mathrm{~m}, 2 \mathrm{H}), 1.46 \sim$ 1.55 (m, 2H), 0.99 (t, $J=7.6 \mathrm{~Hz}, 3 \mathrm{H})$.

4-甲氧基苯甲酸正丁酯(2h) ${ }^{[12]}$ : ${ }^{1} \mathrm{H}$ NMR (400 MHz, $\left.\mathrm{CDCl}_{3}\right) \delta: 7.99(\mathrm{~d}, J=8.8 \mathrm{~Hz}, 2 \mathrm{H}), 6.91$ (d, $J=8.8 \mathrm{~Hz}$, $2 \mathrm{H}), 4.27(\mathrm{t}, J=6.8 \mathrm{~Hz}, 2 \mathrm{H}), 3.85(\mathrm{~s}, 3 \mathrm{H}), 1.69 \sim 1.79(\mathrm{~m}$, $2 \mathrm{H}), 1.45 \sim 1.54(\mathrm{~m}, 2 \mathrm{H}), 0.98(\mathrm{t}, J=7.2 \mathrm{~Hz}, 3 \mathrm{H})$.

4-硝基苯甲酸-2-戊酯(2i) ${ }^{[13]}:{ }^{1} \mathrm{H} \mathrm{NMR}\left(\mathrm{CDCl}_{3}, 400\right.$ MHz) $\delta: 8.28$ (d, $J=8.4 \mathrm{~Hz}, 2 \mathrm{H}), 8.20(\mathrm{~d}, J=8.8 \mathrm{~Hz}, 2 \mathrm{H})$, $5.16 \sim 5.25(\mathrm{~m}, 1 \mathrm{H}), 1.71 \sim 1.81(\mathrm{~m}, 1 \mathrm{H}), 1.58 \sim 1.66(\mathrm{~m}$, $1 \mathrm{H}), 1.38 \sim 1.48(\mathrm{~m}, 2 \mathrm{H}), 1.37(\mathrm{~d}, J=6.0 \mathrm{~Hz}, 3 \mathrm{H}), 0.96(\mathrm{t}$,
$J=7.2 \mathrm{~Hz}, 3 \mathrm{H})$.

4-氯苯甲酸-2-戊酯 $(\mathbf{2} \mathbf{j})^{[14]}$ : ${ }^{1} \mathrm{H}$ NMR (400 MHz, $\left.\mathrm{CDCl}_{3}\right) \delta: 7.97(\mathrm{~d}, J=8.4 \mathrm{~Hz}, 1 \mathrm{H}), 7.40(\mathrm{~d}, J=8.4 \mathrm{~Hz}$, $2 \mathrm{H}), 5.10 \sim 5.20(\mathrm{~m}, 1 \mathrm{H}), 1.66 \sim 1.78(\mathrm{~m}, 1 \mathrm{H}), 1.52 \sim 1.64$ $(\mathrm{m}, 1 \mathrm{H}), 1.36 \sim 1.47(\mathrm{~m}, 2 \mathrm{H}), 1.33(\mathrm{~d}, J=6.0 \mathrm{~Hz}, 3 \mathrm{H})$, $0.94(\mathrm{t}, J=7.2 \mathrm{~Hz}, 3 \mathrm{H})$.

4-甲氧基苯甲酸-2-戊酯(2k) ${ }^{[15]}$ : ${ }^{1} \mathrm{H} \mathrm{NMR}\left(\mathrm{CDCl}_{3}\right.$, $400 \mathrm{MHz}) \delta: 8.00(\mathrm{~d}, J=8.4 \mathrm{~Hz}, 2 \mathrm{H}), 6.91(\mathrm{~d}, J=8.4 \mathrm{~Hz}$, $2 \mathrm{H}), 5.08 \sim 5.20(\mathrm{~m}, 1 \mathrm{H}), 3.86(\mathrm{~s}, 3 \mathrm{H}), 1.71 \sim 1.81(\mathrm{~m}$, $1 \mathrm{H}), 1.52 \sim 1.63(\mathrm{~m}, 1 \mathrm{H}), 1.36 \sim 1.49(\mathrm{~m}, 2 \mathrm{H}), 1.32(\mathrm{~d}, J=$ $5.6 \mathrm{~Hz}, 3 \mathrm{H}), 0.94$ (t, $J=7.2 \mathrm{~Hz}, 3 \mathrm{H})$.

4-硝基苯甲酸苯酯 $(2 \mathrm{l}){ }^{[13]}$ : ${ }^{1} \mathrm{H} \quad \mathrm{NMR}\left(\mathrm{CDCl}_{3}, 400\right.$ $\mathrm{MHz}) \delta: 8.40 \sim 8.33(\mathrm{~m}, 4 \mathrm{H}), 7.52 \sim 7.38(\mathrm{~m}, 2 \mathrm{H}), 7.37 \sim$ $7.14(\mathrm{~m}, 3 \mathrm{H})$.

\section{References}

[1] (a) Kröhnke, F. Angew. Chem., Int. Ed. 1963, 2, 225.

(b) Wu, P.; Wang, Q.-F.; Cai, X.-M.; Yan, C.-G. Chin. J. Org. Chem. 2008, 28, 1899 (in Chinese).

(吴萍, 王琦芳, 蔡习美, 颜朝国, 有机化学, 2008, 28, 1899.) (c) Osyanin, V. A.; Osipov, D. V.; Klimochkin, Y. N. J. Org. Chem. 2013, 78, 5505.

[2] Zhao, L.-X.; Moon, Y.-S.; Basnet, A.; Kim, E.-K.; Jahng, Y.; Park, J. G.; Jeong, T. C.; Cho, W.-J.; Choi, S.-U.; Lee, C. O.; Lee, S.-Y.; Lee, C.-S.; Lee, E.-S. Bioorg. Med. Chem. Lett. 2004, 14, 1333.

[3] (a) Yang, Y.; Gao, M.; Zhang, D.-X.; Wu, L.-M.; Shu, W.-M.; Wu, A.-X. Tetrahedron 2012, 68, 7338.

(b) Shang, Y.; Zhang, M.; Yu, S.; Ju, K.; Wang, C.; He, X. Tetrahedron Lett. 2009, 50, 6981.

[4] Wu, P.; Cai, X.-M.; Wang, Q.-F.; Yan, C.-G. Synth. Commun. 2007, 37, 223.

[5] (a) King, L. C. J. Am. Chem. Soc. 1944, 66, 894.

(b) Babcock, S. H.; Nakamura, J. F. I.; Fuson, R. C. J. Am. Chem. Soc. 1932, 54, 4407.

[6] Phillips, W. G.; Ratts, K. W. J. Org. Chem. 1970, 36, 3144.

[7] Weng, S.-S.; Ke, C.-S.; Chen, F.-K.; Lyu, Y.-F.; Lin, G.-Y. Tetrahedron 2011, 67, 1640.

[8] Bao, Y.-S.; Chen, C.-Y.; Huang, Z.-Z. J. Org. Chem. 2012, 77, 8344.

[9] Tian, J.; Gao, W.-C.; Zhou, D.-M.; Zhang, C. Org. Lett. 2012, 14, 3020.

[10] (a) Ram, R. N.; Soni, V. K.; Gupta, D. K. Tetrahedron 2012, 68 , 9068.

(b) Ding, J.-C.; Wu, S.-W.; Cai, C.-H. Chin. J. Synth. Chem. 2000, 8, 184 (in Chinese).

(丁金昌，吴声文，蔡昌慧，合成化学, 2000, 8, 184.)

[11] Iwasaki, T.; Maegawa, Y.; Hayashi, Y.; Ohshima, T.; Mashima, K. J. Org. Chem. 2008, 73, 5147.

[12] Liu, Q.; Li, G.; He J.; Liu, J.; Li, P.; Lei, A. Angew. Chem., Int. Ed. 2010, 49, 3371.

[13] Won, J.-E.; Kim, H.-K.; Kim, J.-J.; Yim, H.-S.; Kim, M.-J.; Kang, S.-B.; Chung, H.-A.; Lee, S.-G.; Yoon, Y.-J. Tetrahedron 2007, 63, 12720 .

[14] Bamoharram, F. F.; Heravi, M. M.; Roshani, M.; Jahangir, M.; Gharib, A. Appl. Catal. A: Gen. 2006, 302, 42.

(Zhao, C.) 